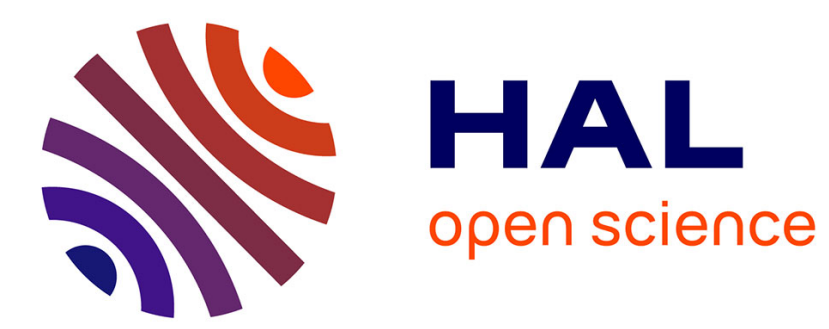

\title{
Un format de partitions interactives
}

Antoine Allombert

\section{To cite this version:}

Antoine Allombert. Un format de partitions interactives. Documents Numériques, 2008, 11 (3-4), pp.29-44. 10.3166/DN.11.3-4.29-44 . hal-00390840

\section{HAL Id: hal-00390840 https://hal.science/hal-00390840}

Submitted on 2 Jun 2009

HAL is a multi-disciplinary open access archive for the deposit and dissemination of scientific research documents, whether they are published or not. The documents may come from teaching and research institutions in France or abroad, or from public or private research centers.
L'archive ouverte pluridisciplinaire HAL, est destinée au dépôt et à la diffusion de documents scientifiques de niveau recherche, publiés ou non, émanant des établissements d'enseignement et de recherche français ou étrangers, des laboratoires publics ou privés. 


\title{
Un format de partitions interactives
}

\author{
Antoine Allombert \\ Laboratoire Bordelais de Recherche en Informatique \\ 351 , cours de la Libération \\ F-33405 Talence \\ IRCAM \\ 1, place Igor Stravinsky \\ F-75004 Paris \\ antoine.allombert@labri.fr
}

RÉSUMÉ. Nous présentons dans cet article un format XML créé pour coder les partitions interactives générées via le système Iscore. Le développement de Iscore est l'aboutissement de travaux de recherche menés au LaBRI en collaboration avec l'Ircam, visant à aboutir à un système de partitions interactives pour la composition et l'interprétation, basé sur des contraintes temporelles. La question du format de sauvegarde des documents édités au travers de ce système s'est rapidement posée et devant les évolutions récentes vers les technologies XML, nous avons décidé d'y répondre par la rédaction d'une DTD (document type definition). Pensé à la base comme support pour le système de sauvegarde de Iscore, ce format pourrait également servir d'interface avec d'autres formats XML et participer à l'élaboration d'un format général de partitions numériques.

ABSTRACT. We present in this paper a XML format used to encode interactive scores created with the Iscore system. This system has been developped at LaBRI in collaboration with Ircam. Its aim is allow the composition and interpretation of interactive scores based on temporal constraints. We faced the question of the saving format by writing a XML DTD. Even if this format was designed only for saving the scores, it will be used as an interface with other XML formats for music and maybe it could be included into general digital score system.

MOTS-CLÉS : composition assistée par ordinnateur, partitions interactives, relations de Allen, $X M L$.

KEYWORDS: computer assisted composition, interactive scores, Allen relations, $X M L$.

DOI:10.3166/DN.11.3-4.29-44 (C) 2008 Lavoisier, Paris

DN - 11/2008. Documents musicaux, pages 29 à 44 


\section{Introduction}

Les recherches qui ont mené au développement du système Iscore partent du constat que les évolutions de la technique et de la musique au $X X^{e}$ siècle ont conduit les compositeurs a créer des pièces sur support (bande magnétique, supports numériques actuellement) non jouables autrement qu'au travers de leur diffusion sur des systèmes d'écoute. Le travail de composition se situe alors dans la fabrication du matériau sonore (synthèse, captation, traitement...) et dans l'organisation des éléments de ce matériau dans le temps pour aboutir à une pièce enregistrée. Ce travail de composition s'effectue de plus souvent au travers d'outils informatiques de plus en plus sophistiqués, mais qui peinent à prendre en compte la dimension de l'interaction avec les pièces lors de l'interprétation de celles-ci par des musiciens. Dans ces conditions la part d'interprétation de ces pièces passe par leur mise en espace lors des concerts. Cette situation a privé une partie des œuvres modernes de l'apport de l'interprétation dont a toujours profité la musique instrumentale. Notre objectif est donc de développer un système permettant l'interprétation de pièces électro-acoustiques au travers des mêmes vecteurs que celle des pièces instrumentales. Partant de là nous avons construit un formalisme permettant de représenter des pièces de musique interactives, ainsi qu'un format de sauvegarde associé.

Nous commençons par référencer quelques travaux autour des formats numériques de documents multimédias dont on peut dans une certaine mesure rapprocher nos partitions. Puis, nous revenons sur le formalisme des partions interactives. Nous présentons ensuite le format de sauvegarde des partitions avant de proposer quelques extensions du modèle et de survoler les champs d'application de notre système.

\section{Discussion autour de l'existant}

Les partitions numériques que nous cherchons à manipuler et donc à représenter au travers de notre format sont constituées d'éléments musicaux (sonores) agencés dans le temps, possédant entre eux des relations temporelles décrites sous forme de relations de Allen et dont le début et la fin peuvent être interactivement déclenchés lors de l'exécution. Par certains aspects, une partition numérique est ainsi comparable à un document multimédia. Cette approche pose la question de la nécessité d'un format particulier pour encoder ces partitions. En effet, plusieurs formats de documents multimédias ont naturellement émergé suite au développement d'applications manipulant ce type de documents. Après l'apparition de formats propriétaires liés à des applications particulières (Com, 1991), des tentatives de standardisation ont vu le jour. Les plus marquantes sont certainement HyTime (HyTime, 1992) et SMIL (SMIL, 2008) qui sont tous deux des formats de type SGML comme l'est notre propre format. Ces deux formats sont intéressants car ils disposent de mécanismes permettant de spécifier des contraintes temporelles entre les éléments qui les constituent et d'autoriser des comportements interactifs. Erfle (1993) montre par exemple comment utiliser le système de dates relatives de Hytime, ainsi que les balises HyOp et HyFunk pour spécifier entre les éléments d'un document des relations de Allen et élaborer des contraintes 
temporelles basées sur des expressions arithmétiques, pour par exemple définir comme constant un intervalle de temps séparant deux événements. L'auteur cite des possibilités d'interaction simple. Notons enfin la présence dans cet article d'une intéressante étude comparative des capacités de quelques formats de l'époque en ce qui concerne l'écriture du temps et de l'interaction. Wirag et al. (1995) utilisent également HyTime pour créer des documents acceptant des modes d'interaction un peu plus élaborés comme des "sauts" entre différentes parties du document, ou encore des situations de choix entre plusieurs scénarios d'exécution du document. Ces efforts présentent des précédents notables de formalisation d'une écriture de la temporalité d'un document multimédia et des possibilités de modifier cette temporalité au cours de la présentation du document. Cependant, si une première approche permet de rattacher des partitions numériques interactives à des documents multimédias interactifs, la spécificité des partitions musicales et les modes d'interaction avec elles méritent que l'on s'attache à produire un formalisme les décrivant ainsi qu'un format associé.

Notre démarche initiale fut de fournir pareil formalisme ainsi qu'une application permettant de manipuler des partitions conforme avec ce formalisme. La création d'un format associé a été rendu nécessaire avec la volonté de sauvegarder les partitions réalisées à l'aide de cet outils. Au-delà de l'intérêt strictement pratique de la sauvegarde des productions des utilisateurs, la description d'un format de partitions interactives nous a paru une bonne occasion d'entamer la réflexion autour de la spécificité de l'interaction musicale par rapport aux documents multimédias plus généraux cités précédemment. Naturellement, notre modèle de partitions encore neuf reste actuellement limité, et de son caractère "simple" peut naître l'idée que les partitions qu'il décrit sont assimilables à des présentations multimédias. On trouvera d'ailleurs au sein de notre formalisme des éléments comme les relations de Allen, également présents dans les documents multimédias. Cependant, la spécificité des partitions se fait déjà sentir. $\mathrm{Au}$ moment de décider du format à adopter pour représenter les partitions, l'idée de se rattacher à un format existant s'est naturellement présentée. Les adaptations nécessaires pour effectuer un tel rattachement qui de surcroît risquaient de s'accroître au fur et à mesure des évolutions de notre formalisme, nous ont poussés à franchir le pas de la création d'un format. Bien sûr, comme nous l'exposons dans la suite, des passerelles vers des formats moins spécifiques sont envisagées.

\section{Modèle théorique}

Nous rappelons ici les éléments théoriques qui sous-tendent notre système. Sans développer totalement notre formalisme de partitions interactives dont le lecteur pourra trouver les détails dans (Allombert et al., 2007), revenons quelques instants sur l'origine de ce travail à savoir la possibilité d'interpréter des pièces de musique électro-acoustique. La question de l'appropriation de pièces électro-acoustiques par des musiciens s'éloignant peu ou prou de la stricte référence au matériau composé reste à ce jour relativement ouverte (Dahan et al., 2008). En effet, à la différence de la musique strictement instrumentale consignée sous forme de partitions symboliques, 
la musique électro-acoustique et en particulier sa composante dite "sur support" ne bénéficie pas des possibilités de modification d'un matériau non plus représenté mais directement enregistré. Plus exactement, certains champs de l'interprétation échappent à des musiciens dont l'intervention lors de la diffusion des pièces est concentrée dans la mise en espace au travers de choix de mixage sur un système d'écoute. Notre motivation initiale a donc été d'étudier la possibilité d'un formalisme de partitions adapté à la pratique des compositeurs et permettant l'interprétation par des musiciens. Pour mettre à jour notre formalisme, nous nous sommes tournés vers l'interprétation de la musique instrumentale pour chercher à en adapter les mécanismes à des types de partitions plus générales.

L'interprétation se différencie de l'improvisation par le fait que les modifications effectuées par le musicien lors de l'exécution sont encadrées par des indications du compositeur. Par conséquent, les libertés proposées au musicien ne sont pas infinies. Pour caractériser ces libertés ainsi que leurs limites, nous nous sommes appuyés sur les travaux de Jean Haury (Haury, n.d.) qui identifie les possibilités pour l'interprétation. Elles sont au nombre de 4 :

- les variations dynamiques, i.e. des variations continues de volume sonore

- l'accentuation qui consiste à modifier très localement le volume

- l'articulation qui correspond à la manière dont les notes vont être liées entre elles

- les modifications agogiques à savoir les décalages des débuts et des fins de note.

Dans le cadre de notre étude, nous ne nous sommes intéressés qu'aux modifications agogiques et donc à la possibilité pour un musicien d'anticiper ou de retarder le déclenchement ou l'arrêt d'une note. Ces modifications sont continuellement à l'œuvre dans la pratique instrumentale, par exemple lors de l'exécution d'un point d'orgue ou lors d'un changement de tempo (accelerando...). Par conséquent, nous avons délaissé temporairement le matériau musical des partitions, pour nous intéresser d'un point de vue temporel au déroulement des éléments qui les constituent. L'hétérogénéité des matériaux sonores impliqués dans les compositions électro-acoustiques rendent le terme de "notes" caduc. Nous considérons donc qu'une pièce est constituée de processus sonores dont dont la mise en œuvre au moment de l'exécution de la pièce va fournir la matière sonore. La nature de ces processus peut être variée, lecture d'un fichier son ou algorithme de synthèse pour citer des exemples simples. Comme nous l'avons dit, les opérations effectuées par ces processus sont en dehors du cadre actuel de notre travail ; nous considérons par conséquent que les éléments constitutifs d'une partition et donc qui sont manipulés par le compositeur sont des représentations symboliques de l'exécution des processus sonores. Comme nous nous intéressons aux modifications agogiques donc à la possibilité de modifier des caractéristiques temporelles (date de début et de fin) des éléments des partitions, les représentations de ces éléments doivent permettre de visualiser et de manipuler ces caractéristiques temporelles. En outre, le travail de composition va se focaliser sur l'écriture du temps, à savoir l'organisation temporelle des éléments de la pièce. Un modèle de représentation adaptée du temps est nécessaire. 


\subsection{Modèle temporel}

Différentes représentations du temps ont été développées dans le cadre des outils informatiques pour la composition assistée par ordinateur. Le lecteur trouvera une introduction à différents modèles temporels ainsi qu'une comparaison entre ceux-ci dans (Desainte-Catherine, 2004). Le choix d'un modèle temporel a répondu à notre volonté d'adapter la pratique de l'interprétation dans la musique instrumentale à la musique électro-acoustique. Jean Haury insiste sur le fait que l'intérêt de l'interprétation réside dans les relations temporelles qu'entretiennent entre elles les notes d'une pièce, c'est-à-dire les possibilités d'enchevêtrement des débuts et fins de notes. Il nous a donc paru important de disposer d'un outil formel permettant de représenter ces relations temporelles. En outre, nous avons préserver les apports des modèles hiérarchiques tel celui utilisé par le logiciel Boxes (Beurivé, 2000). Ce type d'approche permet de tirer parti de différents niveaux hiérarchiques pour exprimer l'organisation de la pièce en structures enchâssées, comme par exemple un découpage en mouvement/partie/mesure/temps. Ainsi, on peut qualifier notre modèle temporel de modèle hiérarchique avec relations temporelles. Dans une première approche, nous avons choisi comme formalisme de relations temporelles les relations de Allen. Ces relations introduites par J.F. Allen en 1983 pour des travaux de linguistique ont été largement utilisées dans différents champs de recherche depuis. Ces relations binaires permettent de décrire l'agencement temporel de deux éléments de durée non nulle. Nous présentons ces relations sur la figure 1.

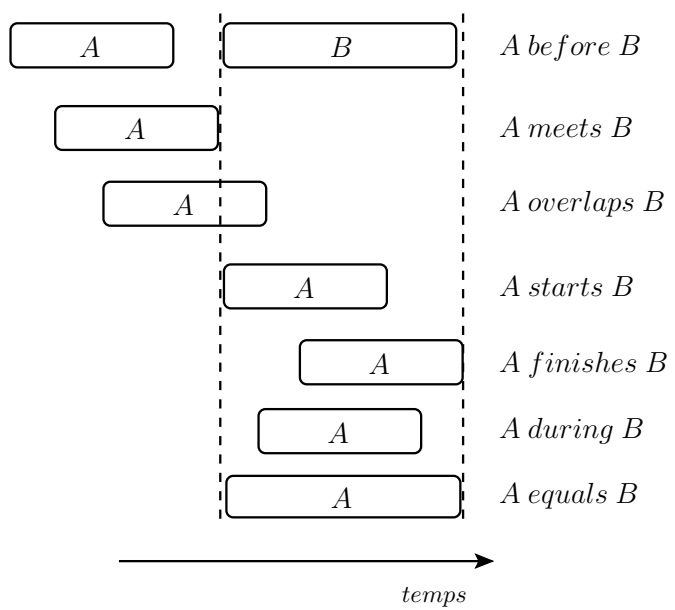

Figure 1. Les relations de Allen

Allen définit à partir de l'ensemble de base et des opérateurs logiques et et ou une grammaire de description temporelle. Pour notre formalisme, nous nous sommes limités à la possibilité de spécifier une seule relation entre 2 objets. Une partition contient donc des objets représentant le déroulement de processus sonores, insérés sur 
des lignes de temps et disposant entre eux de relations de Allen. La multiplicité des lignes de temps est une conséquence de la hiérarchie du modèle. Un objet qui contient d'autres objets (une structure) dispose de sa propre ligne de temps dont l'origine coïncide avec le début de la structure. La spécification des dates de début des objets se fait donc relativement au début de la structure dans laquelle ils sont inclus. La figure 2 présente un exemple de partition composé d'une structure principale $\left(S_{1}\right)$ contenant trois objets : deux objets simples représentant l'exécution de processus $\left(E_{1}\right.$ et $\left.E_{2}\right)$ et une structure $\left(S_{2}\right)$ contenant elle-même un objet simple $\left(E_{3}\right)$. Chaque structure possède sa propre ligne de temps, et les dates de débuts des objets sont relatives au début de la structure qui les contient. Enfin, des relations de Allen overlaps et before ont été définies par le compositeur. Ces relations sont impératives, ce qui signifie que si le compositeur modifie la date de début d'un objet et que la nouvelle date ne respecte pas l'une des relations, le système va calculer des dates pour les autres objets, de telle manière que les relations soient de nouveau respectées. Au cours de la composition, les relations sont une aide au compositeur qui peut définir l'organisation temporelle de sa pièce avant d'ajuster les dates des objets plus précisément en étant sûr que l'organisation générale sera maintenue.

Nous avons utilisé une représentation graphique des objets sous forme de "boîtes" dans la mesure où le matériau musical associé à ces dernières peut être varié. La représentation par boîte à l'avantage d'être suffisamment abstraite pour représenter des objets temporels sans connotation particulière. En outre ces représentations par boîtes permet d'appréhender simplement la hiérarchie. La description de structures complexes regroupant des éléments dans un même ensemble s'effectue de manière naturelle. Notons que l'axe des ordonnées n'a pas de signification, une boîte située au-dessus d'une autre boîte n'a pas de raison de représenter la synthèse d'un son plus aigu par exemple. Comme nous l'avons déjà précisé, la représentation des objets met l'accent sur les caractéristiques temporelles de ces objets à savoir leur début et leur fin, mis en valeur par ce que nous appelons des points de contrôle. Chaque objet a donc deux points de contrôle. Les points de contrôle de l'ensemble des objets d'une partition sont appelés les événements de cette partition.

\subsection{Une touche d'interaction}

Dans le cadre de la musique instrumentale, Jean Haury précise également que le musicien a accès aux possibilités de l'interprétation à travers des points placés dans la pièce qu'il appelle "points d'interaction". Ils s'agit de moments précis où le musicien va pouvoir modifier les paramètres de la partition. Naturellement, on trouve des points d'interaction pour l'ensemble des modalités d'interprétation. Comme nous nous restreignons aux modifications agogiques, nous ne nous sommes intéressés qu'à des points d'interaction permettant de modifier la date d'un point de contrôle (début ou fin d'un objet). On parlera alors d'événement interactif puisque le musicien va pouvoir contrôler explicitement le déclenchement de l'événement au travers du point d'interaction. Un point d'orgue est un bon exemple de point d'interaction pour la musique 


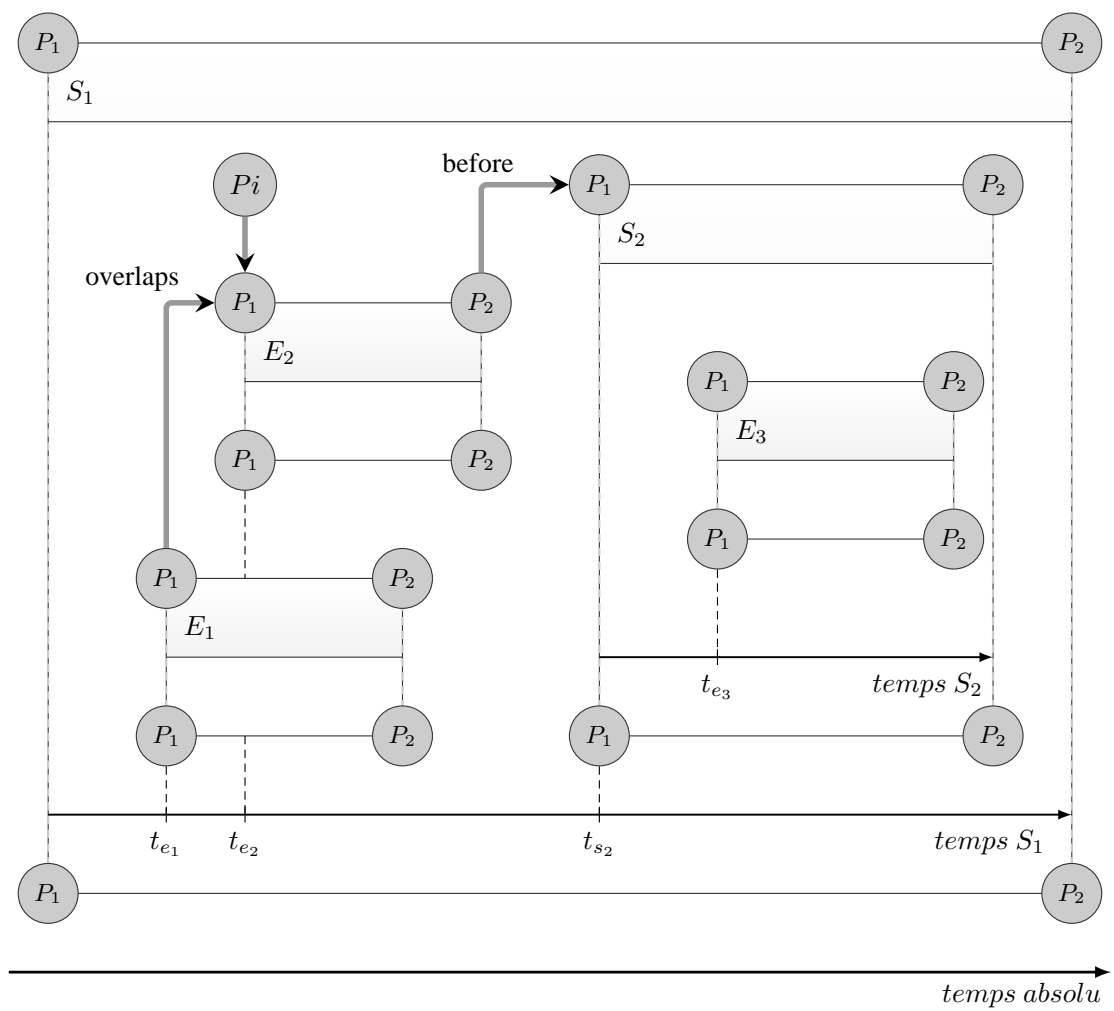

Figure 2. Un exemple de partition interactive

instrumentale dans la mesure où le musicien ou chef d'orchestre peut choisir la durée du point d'orgue et donc de contrôler la fin de la note sur laquelle s'exerce le point d'orgue.

En outre, ces possibilités d'interaction et donc de modification de la pièce s'accompagnent de la définition par le compositeur d'un cadre dans lequel celles-ci vont pouvoir s'exprimer. Dans la musique instrumentale, ce cadre est fixé au travers d'indications du compositeur par exemple de volume ( $p, f f \ldots$ ) ou de tempo (accelerendo...). Ainsi l'interprète est amené à jouir d'un certain nombre de libertés laissées par le compositeur tout en restant dans un cadre fixé par ce même compositeur. Dans le cadre des modifications agogiques et donc à la possibilité de décaler des notes ou d'en modifier la durée par rapport à ce qui est écrit par le compositeur, celui-ci doit être en mesure de définir une organisation temporelle de sa pièce qu'il souhaite voir respectée quels que soient les choix de l'interprète. Les relations de Allen assurent évidemment cette tâche et leur utilité se trouve donc dans les deux phases d'utilisation du système : la composition où elles sont une aide pour le compositeur, et l'interprétation où elles 
définissent le cadre dans lequel l'interprète va exprimer ses libertés. A partir de ce moment la partition écrite ne représente plus que la trame d'une interprétation possible parmi de nombreuses autres, les dates écrites des points de contrôle des objets n'ayant plus de raison d'être strictement respectées. Cependant, lors de l'exécution et de la même manière que lors de la composition, lorsque le musicien modifie la date d'un point de contrôle, le système va recalculer des dates pour les points de contrôles n'ayant pas encore été déclenchés vérifiant d'une part la date choisie par le musicien, et d'autre part les relations de Allen.

Reprenons l'exemple précédent de partition dans laquelle nous rendons le début de $E_{2}$ interactif, en ajoutant un point d'interaction $(P i)$. Par conséquent le musicien va pouvoir anticiper ou retarder $E_{2}$. Mais les deux relations de Allen devant être respectées, en cas de retard sur le début de $E_{2}$, la fin de $E_{1}$ devra être elle-même retardée pour respecter la relation overlaps et le début de $S_{2}$ devra également être retardé pour respecter la relation before. On peut noter qu'il existe une relation de Allen during implicite entre un objet et la structure qui le contient. Par conséquent, l'objet $E_{3}$ sera lui même décalé de part le décalage de l'origine de la ligne de temps de $S_{2}$.

La réaction du système à la modification d'un point de contrôle est pour l'instant limitée à un comportement de type "point d'orgue". Ce qui signifie que pareille modification introduira dans la partition un délais, positif ou négatif, qui va se propager aux points de contrôle succédant le point modifié et ayant un lien avec celui-ci au travers des relations de Allen. Des stratégies de réaction plus élaborées notamment pour modéliser des situations musicales classiques sont à l'étude.

Pour conclure cette présentation, on peut signaler que les modifications temporelles des objets impliquent naturellement des modifications sur les processus dont ils représentent le déroulement. Les processus mis en œuvre doivent donc être capables d'accepter des modifications de la durée de leur exécution et prévoir des stratégies d'adaptation, comme par exemple la génération de valeurs en temps réel si la lecture d'une table à atteint le dernier élément alors que l'exécution nécessite encore des valeurs.

\section{Implémentation}

Il existe actuellement deux implémentations de Iscore l'une développée au Labri et l'autre prenant place dans le système des Maquettes du logiciel de composition assistée par ordinateur de l'équipe Représentations Musicales de l'Ircam OpenMusic(Assayag et al., 1999). Cette dernière version étant la plus aboutie, nous la présentons ici. Les Maquettes sont un environnement spécifique qui se présente sous la forme d'une feuille blanche sur laquelle le compositeur vient placer ses éléments musicaux sous forme de boîtes, l'axe des abcisses représentant la flèche du temps, celui des ordonnées n'ayant pas de signification a priori. Ce formalisme graphique étant en accord avec la représentation de nos partitions, nous avons introduit dans les 


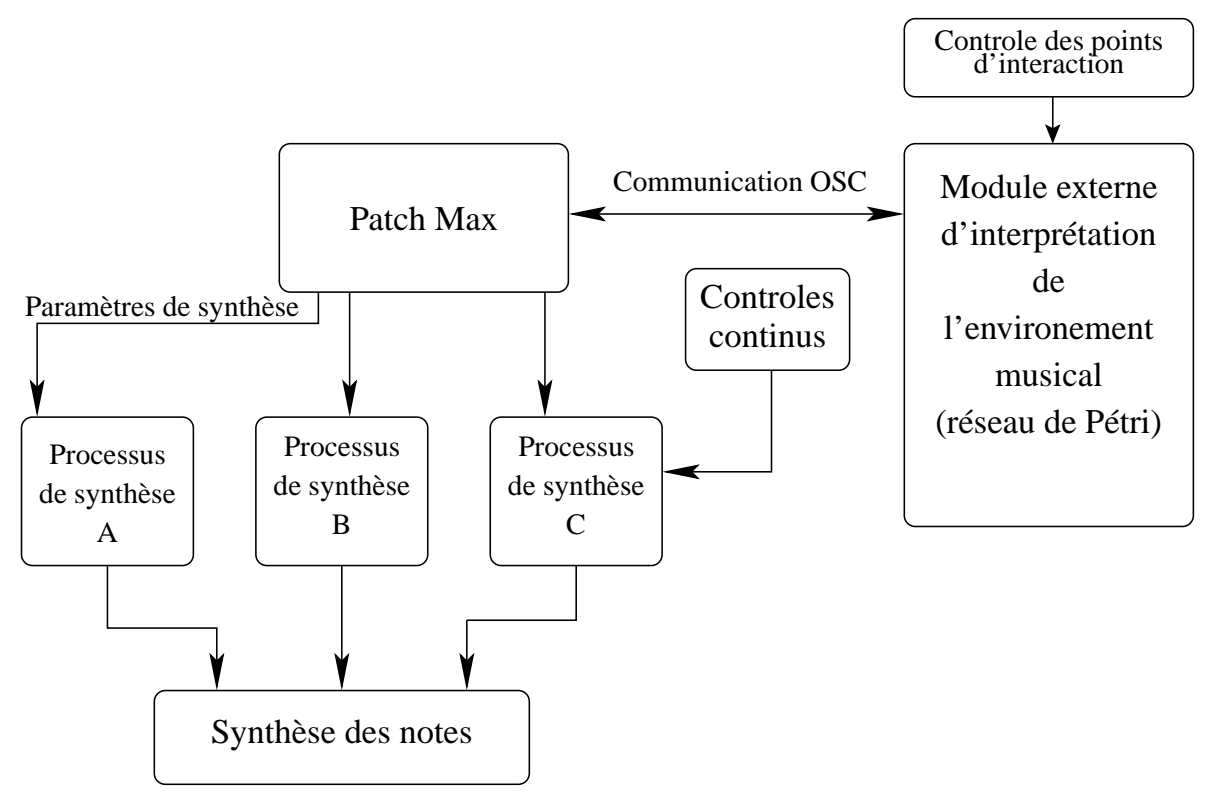

Figure 3. Architecture du système

Maquettes les éléments nécessaires à l'ajout de relations de Allen et de points d'interaction.

Comme nous ne cherchons pas à synthétiser nous même les sons de la pièce, nous utilisons pour ce faire une application extérieure dédiée (typiquement Pure Data) avec laquelle le système va communiquer au travers du protocole Open Sound Control ${ }^{1}$ selon le schéma de la figure 3. L'objectif est donc de déporter la création et la manipulation des processus associés aux objets de la pièce. La conception de ces processus, de telle manière qu'ils soient capables de s'adapter aux modifications temporelles est de la responsabilité du compositeur, aucune vérification à ce sujet n'est opérée par le système. Pour l'exécution de la partition, nous la compilons pour la transformer en réseau de Petri (Diaz, 2001), une structure à états permettant la représentation de processus concurrents devant parfois se synchroniser.

Dans cette version du système un message OSC est associé à chaque événement de la partition, lors de l'exécution de celle-ci, ces messages seront envoyés aux applications concernées lors du déclenchement de chaque événement.

Dans le cadre de cette implémentation de Iscore au sein des Maquettes d'OpenMusic, nous avons dû nous pencher sur les possibilités de sauvegarder les partitions interactives sous la forme de documents indépendants. Du fait que plusieurs implé-

1. http://opensoundcontrol.org/ 


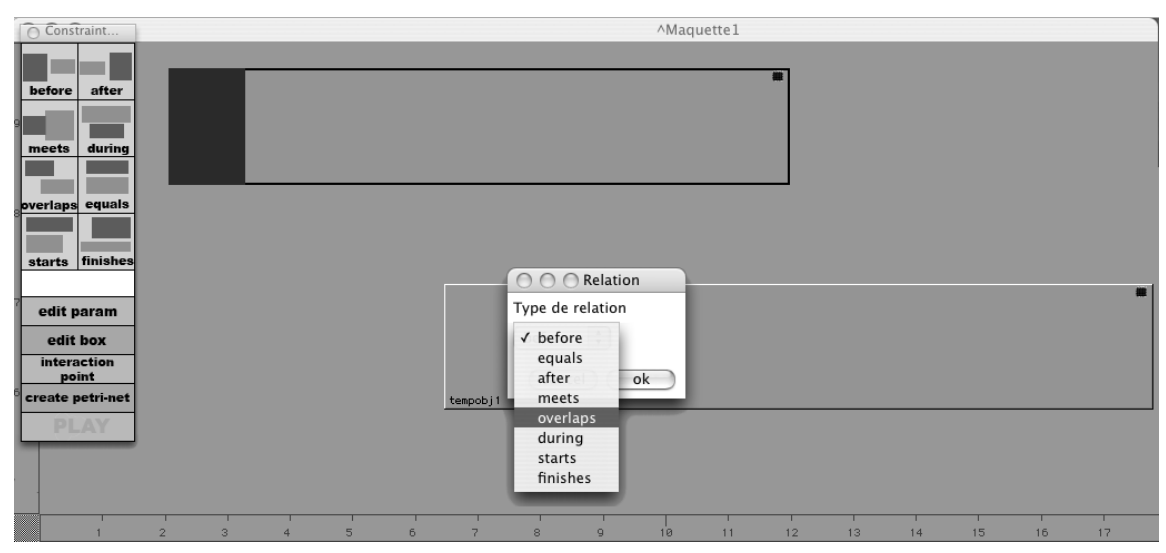

Figure 4. Exemple d'utilisation d'OM pour créer une partition interactive

mentations de Iscore sont amenées à coexister nous nous sommes tourner vers un format indépendant de l'implémentation. L'importance de la hiérarchie dans notre formalisme, ainsi que la popularité des formats de type XML et les facilités de passage entre eux nous ont conduit à écrire une DTD XML.

\section{Format XML}

Notre format ayant pour but premier de sauvegarder les partitions, il contient pour l'instant le strict minimum permettant de décrire une partition interactive. Nous reproduisons ici le fichier résultant de l'export de la partition de la figure 2.

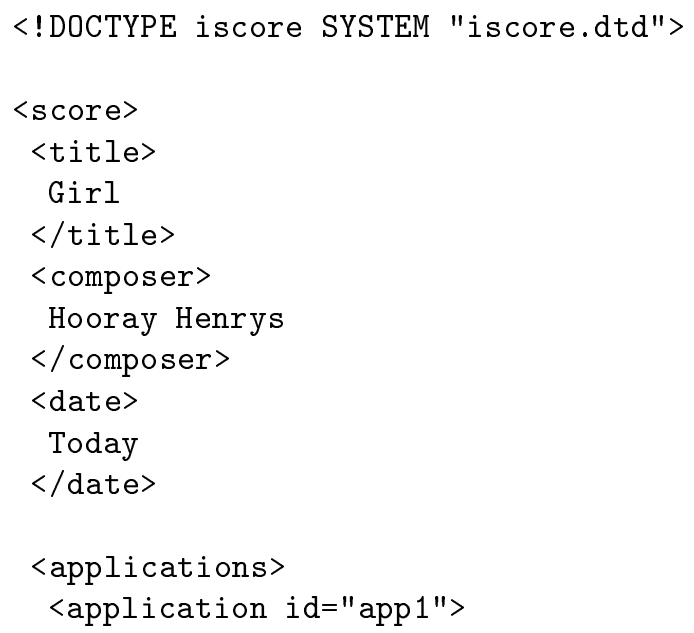




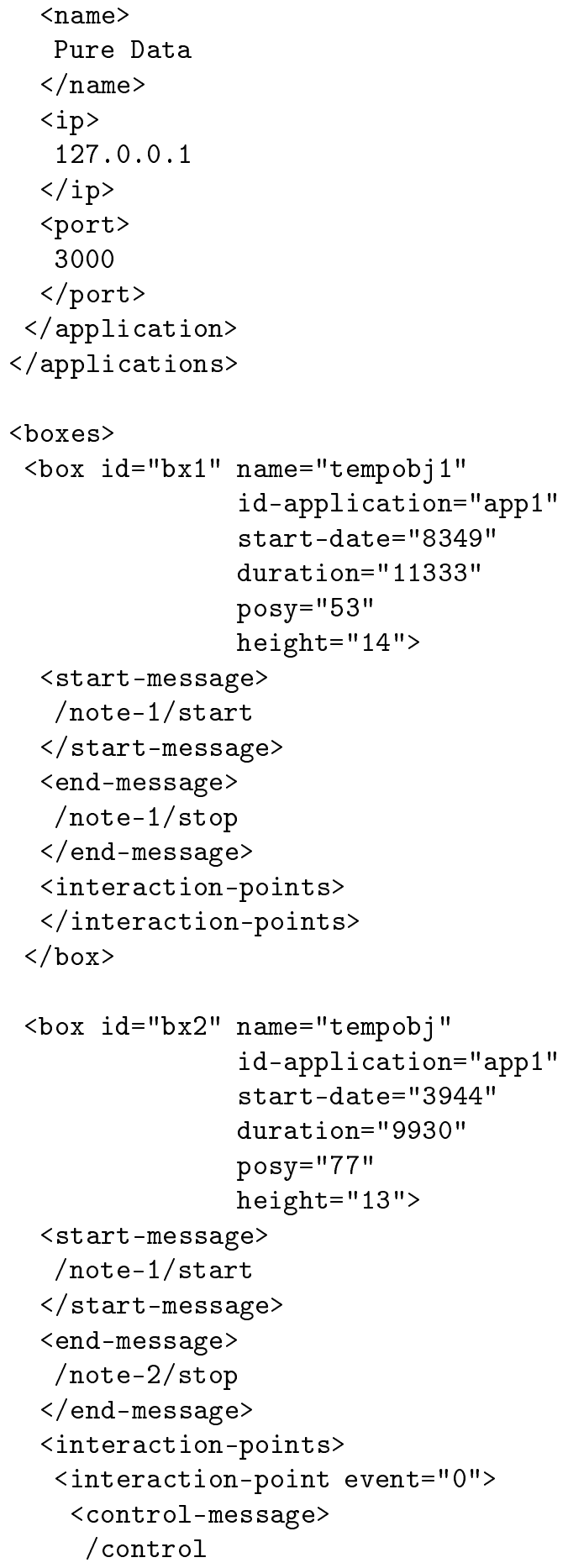




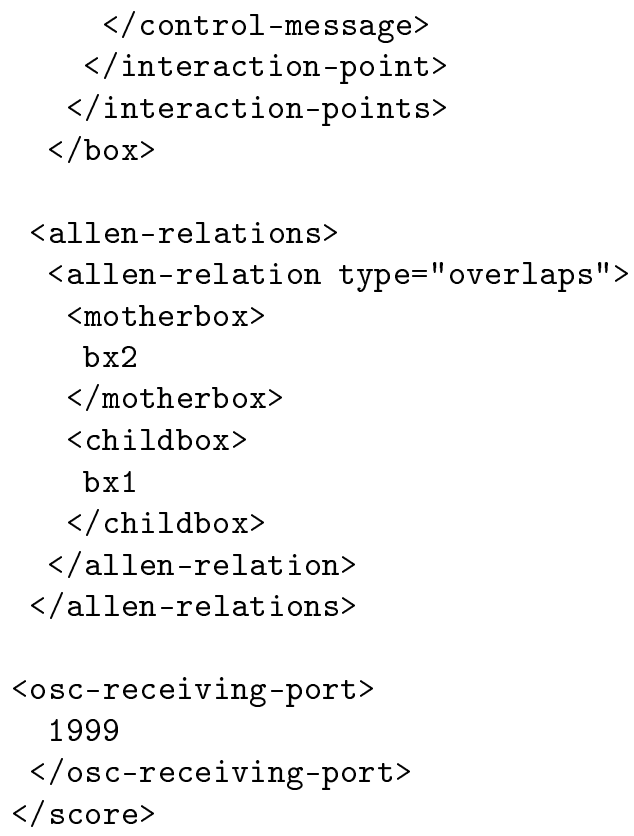

Comme indiqué précédemment, les éléments contenus dans notre format sont simples. La balise racine est la balise <score>. On trouve ensuite quelques informations succinctes sur l'œuvre puis l'ensemble des éléments présents dans la partition sont décrits. La balise <application> permet de coder les informations relatives à une application tiers vers laquelle la partition va envoyer des messages OSC pour exécuter les processus qu'elle utilise Dans notre exemple, une seule application a été définie : Pure Data. Le fichier contient les informations d'adresse réseau permettant à Iscore de communiquer avec cette application. Vient ensuite la liste des boîtes présentes dans la partition. Pour chacune d'entre elle on code leur nom et les informations permettant de les redessiner, comme nous utilisons un système de dates relatives exprimées en fonction de la hiérarchie, les dates de débuts sont données dans le référentiel temporel de la structure qui contient l'objet; cette information est contenue dans la balise <start-date>, tandis que la balise duration contient la durée de la boîte. En outre, on enregistre l'identificateur de l'application chargée d'exécuter le processus dont l'exécution représentée par la boîte, ceci pour permettre l'envoi des messages OSC associés au début et à la fin de la boîte. On trouve bien entendu ces messages OSC dans les balises <start-message > et <end-message>. Les éventuels points d'interactions permettant le déclenchement du début ou de la fin de la boîte sont également présents. Dans notre exemple, l'objet "tempobj" possède un point d'interaction contrôlant son début (event=" 0 "). Dans notre implémentation, ces points d'interaction sont déclenchés au travers de la réception d'un message OSC, cette information est donc présente grâce à la balise <control-message>. On trouve ensuite la liste des relations de Allen avec leur type et les boîtes entre lesquelles elles ont été définies. Enfin, est codé le port d'écoute 
sur lequel le système attendra les messages de déclenchement des points d'interaction (la balise <receiving-port>).

Comme on le voit, ce format est pour l'instant uniquement destiné à recevoir les informations nécessaires à la sauvegarde des partitions telles que décrites pour notre formalisme actuel. Déjà évoquée, sa simplicité actuelle l'apparente à un format de description de documents multimédias généralistes, notons au passage que le protocole OSC est implémenté dans des applications non musicales ce qui permettrait à notre système de manipuler des contenus variés, un projet ${ }^{2}$ est d'ailleurs en cours pour adapter notre formalisme à la régie numérique du spectacle vivant. Cependant, notre formalisme est issu d'une réflexion autour de l'interprétation de pièces de musique. Ainsi l'écriture du temps et de l'interaction s'appuient-t-ils sur des concepts hérités de l'analyse des mécanismes de l'interprétation dans la pratique instrumentale. En particulier, la place centrale qu'occupent les relations temporelles de Allen, ainsi que la possibilité de disposer des points d'interaction sur chaque point de contrôle, différencient notre modèle des formats de documents existants. En outre, le parti pris de l'interprétation à savoir la modification sous contraintes d'un matériau musical composé distingue notre formalisme d'autres travaux autour de la musique interactive.

\section{Perspectives}

Plusieurs pistes s'ouvrent pour les développements futurs de notre système et de son format associé. En ce qui concerne le formalisme lui-même, nous envisageons d'introduire des stratégies de réaction aux modifications du musicien calquant des comportements classiques en musique instrumentale : changement de tempo, rubato... Ces ajouts ancreront encore plus le modèle dans une spécificité musicale.

Concernant le format nous souhaiterions l'étoffer pour lui permettre d'accueillir des informations "annexes" du type de celles concernant le nom du compositeur et de l'œuvre. Une des pistes serait de s'inspirer du format MusicXML ${ }^{3}$ et notamment des manières de coder les pièces "complexes" en utilisant les notions de "parties", "voix"... L'objectif est de compléter notre format pour qu'il devienne réellement un format de codage de partitions musicales comblant une des lacunes des formats actuels, à savoir le codage de la musique électro-acoustique. Cet objectif ambitieux dépasse de loin le cadre de notre étude car pour être complet, un tel format devrait être capable de coder des informations totalement en dehors de notre étude comme par exemple les processus permettant de produire les sons associés aux objets des partitions. Notons que des travaux en ce sens ont déjà été réalisés et citons le langage Faust (Gaudrain et al., 2003) qui permet de décrire des algorithmes impliqués dans des applications musicales, ou encore $\mathrm{MML}^{4}$ langage XML permettant de décrire également des processus de synthèse et de traitement sonores. L'idée serait de s'appuyer sur ces

2. Plate-forme Virage financée par l'ANR.

3. http://www.recordare.com/xml.html

4. Music Markup Language www.musicmarkup.info 
travaux en incluant des parties codées dans ces formalismes pour décrire les processus utilisés dans la pièce.

Une autre voix d'amélioration touche à la question de la représentation graphique des objets et des différents éléments d'une partition. En effet, de nombreux compositeurs ont défini leur propre nomenclature plus ou moins éloignée des représentations traditionnelles. La représentation sous forme de boîtes, même si elle présente de nombreux avantages n'est pas forcément celle retenue dans les systèmes de notation des compositeurs. Une piste serait de permettre la prise en compte de systèmes de notation personnalisés pour les objets. Le format graphique vectoriel $\mathrm{SVG}^{5}$ pourrait être un bon candidat pour coder les systèmes de représentations des compositeurs. Ainsi, un compositeur pourrait produire son propre système de représentation avec des outils de dessin adaptés puis venir appliquer ses motifs sur les boîtes dans Iscore tels des calques pour obtenir la représentation de partition voulue. Le format SVG est également pressenti comme format d'export en vue d'imprimer les partitions ou de les publier sur la toile.

Des possibilités d'export depuis notre format vers des formats de documents multimédias (SMIL par exemple) en retirant les données inadaptées avec le format cible sont envisagées. Cela permettrait éventuellement de publier sur la toile des simulations des partitions.

\section{Applications}

Concernant les utilisations possibles de Iscore, il y a naturellement la création et l'interprétation de pièces de musique électro-acoustique, une pièce est d'ailleurs actuellement en cours de création par Joseph Larralde, assistant musical au Scrime ${ }^{6}$.

Cependant d'autres utilisations sont envisagées comme par exemple un système de play-back qui suivrait le jeu du musicien au travers de points d'interaction. Cette application particulière utiliserait principalement des partitions issues du répertoire instrumental. L'écriture directe d'une partition instrumentale sous forme de boîtes s'avérant assez fastidieuse, nous cherchons à produire directement une partition utilisable par notre système à partir d'une partition existante. Le choix d'un format XML s'avère alors judicieux compte tenu des possibilités de passage entre les divers formats XML. Dans le cadre de cette utilisation, nous cherchons à créer un module de transformation de fichiers Midi et MusicXML en partitions interactives. Ce module devra instancier automatiquement des boîtes et inférer des relations entre elles en fonctions des données du fichier d'entrée. Une dernière utilisation prévue concerne la pédagogie pour permettre à des musiciens débutants de faire l'expérience de l'interprétation de pièce avant de posséder la maîtrise technique pour jouer effectivement la pièce. L'idée est

donc de déléguer une partie des notes au système et de conserver un nombre limité de

5. http://www.w3.org/Graphics/SVG/

6. Studio de Création et de Recherche en Informatique et Musique Electroacoustique http://scrime.labri.fr 
points d'interaction pour contrôler des parties réellement pertinentes pour l'interprétation. D'une manière générale, le système peut permettre d'adapter des pièces aux capacités de jeu des musiciens : débutants, virtuoses, handicapés... Dans ce contexte, le module d'importation de fichiers midi ou musicxml est également central.

\section{Conclusion}

Nous avons présenté dans cet article un formalisme ainsi qu'un format associé permettant de représenter des partitions numériques interactives. Impliquant des objets sonores organisés dans le temps au travers de relations de Allen, ce formalisme se distingue des documents multimedias généralistes par la référence qu'il fait à l'interprétation musicale dans la musique instrumentale. Le format XML associé permet d'une part de sauvegarder les partitions composées dans notre système, mais également de faire l'interface avec d'autres formats. Ces liens avec des formats existants permettraient d'inclure des parties spécifiques codées dans d'autres formats ou de générer automatiquement des partitions interactives à partir de partitions numériques existantes.

\section{Bibliographie}

Allombert A., Assayag G., Desainte-Catherine M., « A System of interactive Scores based on Petri Nets », Pr. of the 4th Sound and Music Computing Conference (2007), Lefkada, Greece, July, 2007.

Assayag G., Rueda C., Laurson M., Agon C., Delerue O., « Computer Assisted Composition at IRCAM : From PatchWork to OpenMusic », Computer Music Journal, 1999.

Beurivé A., « Un logiciel de composition musicale combinant un modèle spectral, des structures hiérarchiques et des contraintes », Journées d'Informatique Musicale, JIM 2000, 2000.

Com, QuickTime Developper's Guide, developer technical publication edn. 1991.

Dahan K., Laliberté M., « Réflexions autour de la notion d'interprétation de la musique électroacoustique », Actes des $13^{\text {ieme }}$ Journées d'Informatique Musicale (JIM 08), Albi, France, March, 2008.

Desainte-Catherine M., « Modèles temporels pour la Synthèse Musicale », in F. Pachet, J. Briot (eds), Informatique Musicale, Hermès, p. 269-309, 2004.

Diaz M. (ed.), Les réseaux de Pétri, Modèles fondamentaux, Hermès, 2001.

Erfle R., " Specification of Temporal Constraints in Multimedia Documents using HyTime », Electronic Publishing, vol. 6, n 4, p. 397-411, 1993.

Gaudrain E., Orlarey Y., A Faust Manual, GRAME. 2003.

Haury J., « La grammaire de l'exécution musicale au clavier et le mouvement des touches », Manuscrit, n.d.

HyTime, HyTime. Information Technology - Hypermedia/Time-based Structuring Language (HyTime). ISO/IEC DIS 10744, 8. 1992.

SMIL, SMIL 3.0 W3C Recommendation. December, 2008. 
44 DN - 11/2008. Documents musicaux

Wirag S., Rothermel K., T.Wahl, « Modelling Interaction with HyTime », Proc. GI/ITG Kommunikation in Verteilten Systemen, p. 188-202, 1995. 